\title{
Omnidirectional mobile robot platform with four Mecanum wheels featuring remote motion control through either a graphical application or an inertial measurement unit
}

\author{
Carlos Arantes ${ }^{1[0000-0003-0458-0578]}$ and João Sena Esteves ${ }^{1,2[0000-0002-3492-1786]}$ \\ ${ }^{1}$ Department of Industrial Electronics, ${ }^{2}$ Algoritmi Research Centre \\ University of Minho, Campus of Azurém, 4800-058 Guimarães, Portugal \\ a58765dalunos.uminho.pt, senaldei.uminho.pt
}

\begin{abstract}
Omnidirectional mobile platforms allow simultaneous translation and rotation, which leads to the optimization of their trajectories, a possible reduction of the distance traveled and, consequently, a reduction of the energetic consumption. This paper presents an omnidirectional mobile robot platform based on four Mecanum wheels, a graphical application which allows remote motion control and monitoring of several parameters of the platform, and an inertial remote control that uses an IMU (Inertial Measurement Unit) and an AHRS (Attitude and Heading Reference System) to set the movements of the platform.
\end{abstract}

Keywords: Mecanum wheels, omnidirectional mobile platform, motion control, AHRS, IMU.

\section{Introduction}

In many applications, the use of non-omnidirectional platforms constitutes an important limitation. An example is a classic electric wheelchair such as the one presented in [1]. Its main purpose is to facilitate the transportation of disabled people but it cannot perform some movements in an $x O y$ plane because it uses the Ackermann steering system, which has 2 DOF (degrees of freedom). Usually, non-omnidirectional steering systems require complex algorithms for the dynamic management of trajectories and they may imply large distances traveled, a waste of energy and high response times.

Omnidirectional mobile platforms allow simultaneous translation and rotation, which leads to the following benefits:

1) Optimization of the trajectories of the mobile platform - The distance traveled is minimized due to the combination of simultaneous rotation and translation;

2) Possible reduction of the energetic consumption - Since the distance traveled is minimized, the required energy may also be decreased.

This is a pre-copyedited version of a contribution published in Innovation, Engineering and Entrepreneurship (Editors: José Machado, Filomena Soares and Germano Veiga), published by Springer, Cham. The definitive authenticated version is available online via https://doi.org/10.1007/978-3-319-91334-6_5. 
Mobile platforms with four Mecanum wheels [2][3] are omnidirectional. Each wheel has its own motor and platform motion control requires controlling each motor individually. The great variety of movements achievable through simultaneous translation and rotation cannot be properly controlled by traditional steering wheels used in non-omnidirectional platforms. More adequate solutions have been developed, usually based in joysticks [4][5]. A motion control system based in hand gestures recognition is presented in [6].

This paper presents an omnidirectional mobile robot platform based on four Mecanum wheels (which is inherently more stable than those with only three wheels), a graphical application which allows remote motion control and monitoring of several parameters of the platform, and an inertial remote control that uses an IMU (Inertial Measurement Unit) and an AHRS (Attitude and Heading Reference System) to set the movements of the platform. The complete system is suitable, for example, for educational purposes.

\section{System Architecture Overview}

The developed system (Figure 1a) has three main components, which will be detailed in this section: 1) Omnidirectional mobile robot platform, 2) Graphical application, and 3) Remote control.

\subsection{The Omnidirectional Mobile Robot Platform (OMRP)}

The omnidirectional mobile robot platform (OMRP) exchanges data either with the graphical application or the remote control. As shown in Figure 1a, it has several subsystems. The power supply subsystem, which has both ultracapacitors (they allow ultrafast charging) and a conventional lead-acid battery (for increased autonomy) as energystoring devices, provides the required electrical power for each part of the OMRP. It is detailed on [7]. The OMRP has four Mecanum wheels placed as suggested in Figure $1 b$. For a two-dimensional $x O y$ plane, its steering system provides three independent possible movements: a translation along the $\mathrm{x}$-axis, a translation along the $\mathrm{y}$-axis and a rotation about the z-axis. 


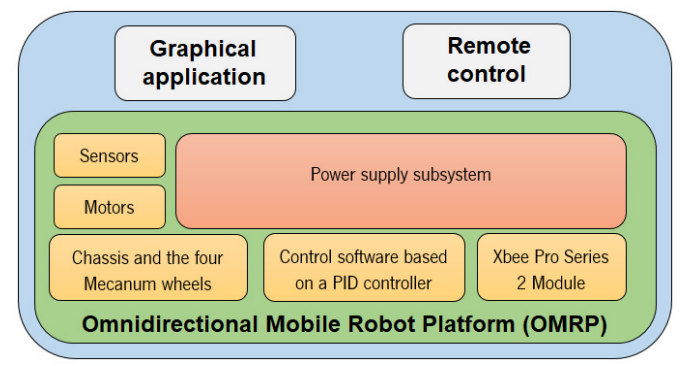

a)

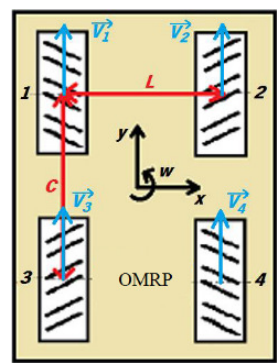

b)

Fig. 1. a) Block diagram of the developed system; b) Schematic top view of the OMRP, clarifying the positions of the four Mecanum wheels and the referential used.

Controlling the movements of the OMRP requires defining its instantaneous velocity, which may be given as a three-dimensional vector: the linear velocity in the $\mathrm{x}$-axis $v_{x}$, the linear velocity in the y-axis $v_{y}$ and the angular velocity about the z-axis $w_{z}$. Taking this into consideration, it is necessary to compute the instantaneous velocity of each Mecanum wheel. Such computation requires the following conversion model that specifies the relationship between the velocities $v_{x}, v_{y}$ and $w_{z}$, and the velocity of each Mecanum wheel, $v_{1}, v_{2}, v_{3}$ and $v_{4}(C$ and $L$ are the distances highlighted in Figure $1 \mathrm{~b}$; International System units are considered) [8] [9] [10]:

$$
\left[\begin{array}{l}
v_{1} \\
v_{2} \\
v_{3} \\
v_{4}
\end{array}\right]=\left[\begin{array}{ccc}
1 & 1 & -\frac{C+L}{2} \\
-1 & 1 & \frac{C+L}{2} \\
-1 & 1 & -\frac{C+L}{2} \\
1 & 1 & \frac{C+L}{2}
\end{array}\right] \cdot\left[\begin{array}{l}
v_{x} \\
v_{y} \\
w_{z}
\end{array}\right]
$$

A PID controller system was used in order to ensure that the measured velocity is as close as possible to the computed reference velocity. The PID controller has four instances, each one matching one Mecanum wheel. An anti-reset windup block was also implemented into the PID controller in order to minimize the risk of high overshooting on its output. Finally, a wireless module was integrated on the OMRP, enabling it to communicate with either the graphical application or the remote control. It was set a peer-to-peer network based on XBee Pro Series 2 modules [11].

\subsection{The Graphical Application}

The graphical application is a program, written in C\# programming language (running on a .NET virtual machine), used to wirelessly control the movements of the OMRP using a personal computer. It also provides remote monitoring of some parameters of the OMRP. It was designed in order to have two main windows. The main goal of the 
first one is to establish the wireless communication between the OMRP and the computer that runs the graphical application. When the graphical application is used, an XBee Pro Series 2 module must be connected to the computer so as to establish the communication. Another module is installed on the OMRP. The two modules establish the wireless network by themselves. On the computer side, a user simply has to select the serial port that matches the modules connected to the computer. When the right serial port is selected, some frames are exchanged in order to verify the state of the wireless connection. If no error occurs, the second window opens. Otherwise, an error message is shown and the second window is not opened.

The second window aims to provide a way for both OMRP movements definition and monitoring of the OMRP. It was designed to have four separators in order to keep the data and settings organized. The first separator provides real-time information about the status of the wireless connection, some electrical parameters of the power supply subsystem of the OMRP, the environment temperature and humidity. The second separator provides feedback about the performance of the PID controller that manages each of the four Mecanum wheels. The separator shows in real-time the reference velocity, the measured velocity and the error (given by the difference between the reference velocity and the measured velocity). This feedback is provided using real-time graphs. The third separator offers the possibility of controlling the movements of the OMRP. The design of the separator was done having into account that it should have an intuitive interface and, at the same time, it should allow the execution of any trajectory. To achieve that, a set of buttons was created, each one to set a standard movement of the OMRP. Furthermore, a set of three numerical input boxes was designed to ensure the possibility of defining the velocities $v_{x}, v_{y}$ and $w_{z}$ independently. The fourth separator provides some independence to the OMRP movements. In other words, the graphical application should be able to command the OMRP in order to ensure that it performs some autonomous and useful movements. Two operation modes were considered in the implementation. The first one consists in following an object autonomously - using its infrared sensors, the OMRP follows an object trying to keep a constant distance to it. In the second operation mode, the OMRP autonomously tries to maintain a constant distance to the wall while moving forward or backward at a certain speed.

\subsection{The Remote Control}

The remote control provides an intuitive way of wirelessly controlling the movements of the OMRP, using an IMU. An IMU has, at least, an accelerometer and a gyroscope. The remote control uses a 9-DOF IMU Pololu MinIMU-9 V2. It contains a 3 DOF accelerometer, a 3 DOF gyroscope and a 3 DOF compass. Using the data given by the three sensors and the DCM (Direction Cosine Matrix) algorithm [12], it is possible to set up an AHRS, a system that collects the data from an IMU and converts it to a new referential whose origin is the centre of mass of the remote control. Its rotation axes are roll, pitch and yaw. The computed values of roll, pitch and yaw must be converted into the three velocity components of the OMRP $\left(v_{x}, v_{y}\right.$ and $\left.w_{z}\right)$, which is accomplished through the model 


$$
\left[\begin{array}{l}
v_{x} \\
v_{y} \\
w_{z}
\end{array}\right]=\left[\begin{array}{lll}
0.8 & 0.8 & 1
\end{array}\right] \cdot\left[\begin{array}{c}
\text { roll } \\
\text { pitch } \\
y a w-y a w_{0}
\end{array}\right]
$$

where the angles of the AHRS (roll, pitch and yaw) are given in degrees, linear velocities are given in $\mathrm{cm} / \mathrm{s}$ and angular velocity is given in $\mathrm{rad} / \mathrm{s}$. Angle $y a w_{0}$ is a reference heading and $y a w_{l}$ is the actual heading. The gains $0.8,0.8$ and 1 were obtained empirically and they set the sensitivity of the movements of OMRP with respect to the AHRS. Besides the IMU, the remote control incorporates an XBee Pro Series 2 module and an Atmel Atmega $328 P$ microcontroller for handling the collected data through the DCM algorithm. The system is powered by a $9 \mathrm{~V}$ rechargeable Ni-MH battery. A block diagram of the developed remote control is shown in Figure 2.

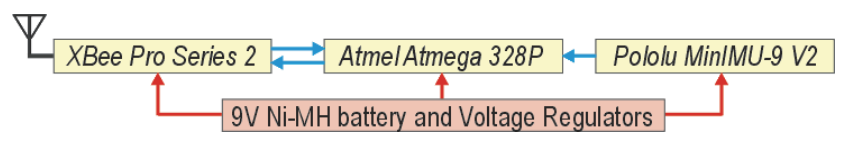

Fig. 2. Block diagram of the developed remote control.

\section{Results}

This section presents some results obtained with the previously described system.

\subsection{The Omnidirectional Mobile Robot Platform (OMRP)}

Figure 3 shows the final aspect of the developed OMRP and the prototype of the remote control. Coupled to each wheel of the OMRP there is a gearbox, an optical encoder and a brushed DC motor. In order to measure the distance to objects and obstacles, the OMRP also has four analog infrared sensors Sharp GP2Y0A21YK0F [13] (one at each side of the platform).

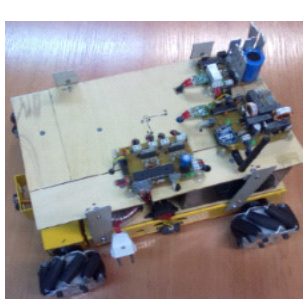

a)

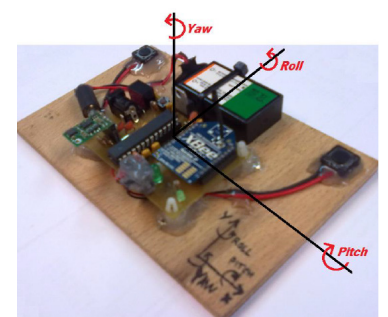

b)

Fig. 3. a) Final aspect of the developed OMRP; b) Prototype of the remote control.

The OMRP was tested. It is capable of exchanging data with either the graphical application or the remote control. Each Mecanum wheel can be controlled independently, enabling the platform to perform any movement on an $x 0 y$ plane. A video demonstrating the OMRP operating may be found here:

https://www.youtube.com/watch?v=Nm4K14rrIr8\&feature=youtu.be. 
It was validated that the OMRP is capable of following a moving body, keeping a predefined constant distance to it. It was also tested that it is able to maintain a predefined constant distance to a wall while moving forward or backward at a specific speed.

\subsection{The Graphical Application}

The first window of the developed graphical application enables a user to select the right serial port of the computer in order to start the connection with the OMRP. Then, the second window comes up evidencing its four separators. The first separator shows the values of several parameters of the OMRP, such as electric output power, ultracapacitors voltage and environment temperature. The second separator presents graphs related to the speed of each Mecanum wheel (Figure 4a) - the green line is the reference speed, the blue line is the real speed and the red line is the error (difference between reference speed and real speed). The third separator enables a user to command the OMRP through a set of intuitive buttons (Figure 4b). Finally, the fourth separator is focused on enabling the OMRP to follow an object autonomously (keeping a specific distance to it) or to maintain a constant distance to the wall while moving forward or backward at a certain speed.

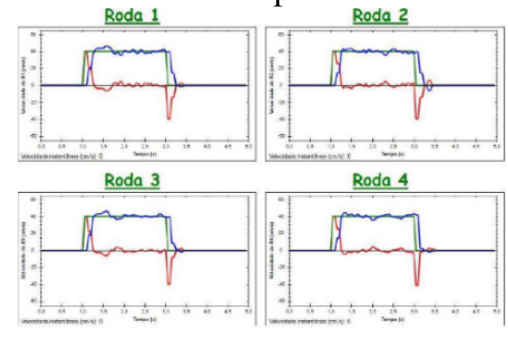

a)

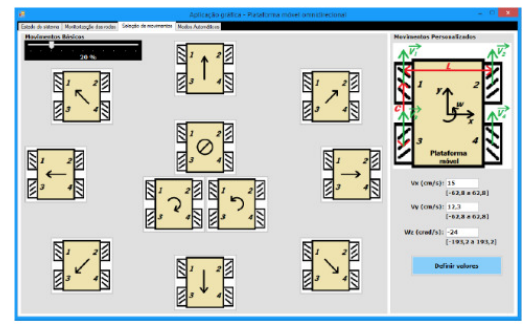

b)

Fig. 4. a) Second separator of the second window of the graphical application; b) Third separator of the second window of the graphical application.

\subsection{The Remote Control}

The prototype of the remote control was developed and tested. It is capable of computing its values of roll, pitch and yaw. Two buttons were added to ensure safety - data is only sent to the OMRP while both buttons are pressed simultaneously. It was found that the computed values of the velocities $v_{x}, v_{y}$ and $w_{z}$, sent to the OMRP, may be updated at a frequency of $50 \mathrm{~Hz}$, considering a system clock of $16 \mathrm{MHz}$. The energy consumption of the remote control was measured. It needs $585 \mathrm{~mW}$ of electric power and its $9 \mathrm{~V}$ battery lasts for, approximately, 1 hour and 13 minutes.

\section{Conclusions and Future Developments}

This paper presented an omnidirectional mobile robot platform (OMRP) with four Mecanum wheels. It is capable of 1) following a moving body, keeping a constant distance to it, and 2) maintaining a constant distance to a wall while moving forward or 
backward at a specific speed. The motion control of the platform is also possible through the use of either a graphical application or a remote control.

Besides controlling the movements of the OMRP, the graphical application monitors some parameters of both the environment and the platform, including showing the performance of the PID controller that tries to keep the measured velocity of the Mecanum wheels as close as possible to a reference velocity. This application is useful in order to study how the error (difference between the reference velocity and measured velocity) affects the trajectory described by the mobile platform.

The developed system may be used for educational purposes in the scope of omnidirectional platforms based on Mecanum wheels and AHRS. Furthermore, since the OMRP has the advantages inherent to omnidirectional platforms in terms of freedom of movements, it may be used in places in which the space is reduced.

As future developments, it is suggested to add a LIDAR sensor in order to enable the OMRP to have a very precise mapping of the space around it. Adding sensors for detecting harmful gases such as carbon monoxide would also be useful in some applications.

\section{Acknowledgements}

This work has been supported by COMPETE: POCI-01-0145-FEDER-007043 and FCT - Fundação para a Ciência e Tecnologia within the Project Scope: UID/CEC/00319/2013.

\section{References}

1. Christina Tsalicoglou, Xavier Perrin, "Survey on Navigation Assistants for People with Disabilities", Autonomous Systems Laboratory, ETHZ, Zurich, Switzerland, 2010.

2. R.P.A. van Haendel, "Design of an omnidirectional universal mobile platform", DCT 2005.117, DCT traineeship report, Eindhoven, September 2005.

3. Onib Nasir, Musman Yousuf, "Introducing: The Mecanum Wheel", IEEE PNEC PERSPECTIVE, Vol. 4, Autumn 2012.

4. Luís Sarmento, Francisco Nunes, Ricardo Santos Martins, João Sepúlveda and João Sena Esteves; "Remote Control System for a Mobile Platform with Four Mecanum Wheels"; International Journal of Mechatronics and Applied Mechanics, Volume 2017, Issue 1, Pages 274-281. Cefin Publishing House, 2017. ISSN: 25596497; EID: 2-s2.0-85029820801

5. M. K. S. H. Maldeniya, R. C. Madurawe, L. B. H. T. Thilakasiri, T. M. S. Thennakoon, R. M. T. P. Rajakaruna, "Remote Controlled 4WD Omni Directional Robot Using Mecanum Wheels"; Department of Mechatronics, Faculty of Engineering, South Asian Institute of Technology and Medicine (SAITM), Sri Lanka, 2015.

6. Rui Vilaça, Jorge Ramos, João Sepúlveda and João Sena Esteves; "Mobile Platform Motion Control System Based on Human Gestures"; International Journal of Mechatronics and Applied Mechanics, Volume 2017, Issue 1, Pages 267-273. Cefin Publishing House, 2017. ISSN: 25596497; EID: 2-s2.0-85029814404

7. Carlos Arantes, João Sena Esteves, João Sepúlveda, "A new energetically optimized power supply system for a mobile robot platform, using batteries and ultracapacitors to ensure both 
ultrafast charging and autonomy", ICINCO 2015, 12th International Conference on Informatics in Control, Automation and Robotics, 2015.

8. Ryan Thomas, "Omni-Directional Mobile Platform for The Transportation of Heavy Objects", Massey University, Palmerston North, New Zealand, 2011.

9. Christof Rohrig, Daniel Heb, Christopher Kirsch, Frank Kunemund, "Localization of an Omnidirectional Transport Robot Using IEEE 802.15.4a Ranging and Laser Range Finder", The 2010 IEEE/RSJ International Conference on Intelligent Robots and Systems, Taipei, Taiwan, October 18-22, 2010.

10. Jungmin Kim, Jungje Park, Sungshin Kim, "Inertial Navigation System for Omni-directional AGV with Mecanum Wheel", School of Electrical Engineering, Pusan National University, Geumjeong, Busan 609-735, Korea, Advances in Mechanical Engineering, ISSN: 21600619, Vol. 2, No. 1, March 2012.

11. Digi International, "XBee®/XBee-PRO® ZB RF Modules", 11001 Bren Road East, Minnetonka, MN 55343, November 2010.

12. Erick Macias, Daniel Torres, Sourabh Ravindran, "Nine-Axis Sensor Fusion Using the Direction Cosine Matrix Algorithm on the MSP430F5xx Family", Texas Instruments, Application Report, SLAA518A - February 2012.

13. Sharp Corporation, "GP2Y0A21YK0F", Datasheet, December 2006. 